\title{
Efficient one-step melt-compounding of copolyetheramide/pristine clay nanocomposites using water-injection as intercalating/exfoliating aid
}

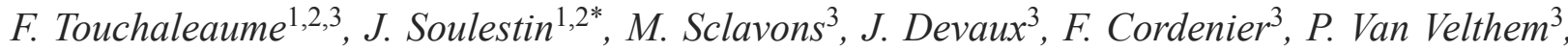 \\ J. J. Flat ${ }^{4}$, M. F. Lacrampe Li,2, P. Krawczak $^{1,2}$ \\ ${ }^{1}$ Univ. Lille Nord de France, F-59000 Lille, France \\ ${ }^{2}$ Ecole des Mines de Douai, Department of Polymers and Composites Technology \& Mechanical Engineering, 941 \\ Rue Charles Bourseul, BP 10838, F-59508, Douai, France \\ ${ }^{3}$ Institute of Condensed Matter and Nanoscience (Bio- and Soft-Matter) \& Ecole Polytechnique de Louvain, Université \\ Catholique de Louvain, Croix du Sud, 11348 Louvain-la-Neuve, Belgium \\ ${ }^{4}$ Arkema - Cerdato, F-27470 Serquigny, France
}

Received 13 April 2011; accepted in revised form 4 July 2011

\begin{abstract}
Polyether-block-amide (PEBA) /clay nanocomposites were prepared water-assisted by twin-screw extrusion. Both organomodified and pristine (i.e. purified but non-modified) montmorillonite clays were used. A high-pressure differential scanning calorimetry analysis carried out in the processing conditions demonstrated that PEBA/water blend exhibits some miscibility and that amide blocks and water behave as a single phase. In addition to a significant decrease of the melting temperature, water injected into the melt plays a key role among the filler dispersion and prevents the matrix from degradation during melt-extrusion. This process enables the compounding of pristine clay-based nanocomposites whose dispersion state is high enough for the resulting mechanical performances in tension to be at least equivalent to what is reached with organomodified clay. Effects of the nanofiller dispersion onto the macromolecules' mobility are detailed and fracture mechanisms are identified for the various structures.
\end{abstract}

Keywords: nanocomposites, poly(ether-block-amide), pristine clay, water injection

\section{Introduction}

Polyamide/clay 'hybrids' were seen as pioneer materials 20 years ago among the polymer nanomaterials community [1]. Henceforth, different kind of polyamides (PA) or polyamide copolymers have been identified as suitable matrices for the elaboration of polymer/clay nanocomposites [2-5], bearing favorable polar amide functions along the backbone. Most of the studies dealing with PA/clay nanocomposites recourse to organically modified clay to successfully disperse the nanoplatelets. However, polyamide shows better thermodynamical interac- tions with pristine clay than with modified clay as calculated by molecular simulation $[6,7]$ and illustrated by reported successful (i.e. dispersed and exfoliated) PA6/pristine clay nanocomposites elaboration by in situ polymerization [8]. An interaction mechanism has been proposed by Shelley et al. [2] who mention the complexation of the polyamide chains (precisely by the carbonyl oxygen atoms) with the negatively charged layers. This complex distorts the conformation of the backbone at the vicinity of the mineral, explaining the widely reported crystalline phase modification in PA6/montmoril-

\footnotetext{
${ }^{*}$ Corresponding author, e-mail: jeremie.soulestin@mines-douai.fr
} (c) BME-PT 
lonite (MMT) nanocomposites [9]. The emergence of gamma phase is representative of the favorable polar/polar interactions existing between polyamide 6 and clay. Nevertheless, a substantive role played by alkylammonium ions during melt blending is the layers preexfoliation, the platelets needing to be initially intercalated for the macromolecules to enter the galleries and to exfoliate the mineral sheets. However at the processing temperatures of polymers such as polyamides, those surfactants suffer thermal degradation [10] leading silicate layers to collapse which limits the clay dispersion. Alternative ways to preexfoliate the clay layers (generally montmorillonite) have been investigated but induce also an extra cost. The interlayer distance can be increased either before melt blending the filler into the matrix by exchanging the initial $\mathrm{Na}^{+}$counterion with a larger inorganic cation $\left(\mathrm{K}^{+}, \mathrm{Ca}^{2+}, \mathrm{Mg}^{2+}, \mathrm{Ba}^{2+}\right.$ ...) $[11,12]$, ionic liquids $[13,14]$, by treating the mineral with a cold-plasma in order to graft hydrocarbon compounds on its surface [15] or by supercritical $\mathrm{CO}_{2}$-assisted process $[16,17]$. In the latter process, $\mathrm{CO}_{2}$ molecules are claimed to penetrate the interlayer spacing in the supercritical state, a further depressurization makes the fluid expand and clay layers separate from each other.

Furthermore, due to their highly polar amide functions, polyamides are hydrophilic materials. PA water solubility under temperature and pressure conditions has widely been reported in the literature [18-24]. Considering this peculiar behavior of polyamides and that montmorillonite platelets are separated from each other in water (constituting a colloidal dispersion), attempts have been made to elaborate PA/pristine clay nanocomposites by melt extrusion with the aid of water. Two different approaches have been proposed: injection of a slurry (clay dispersed into water) into the melt [25] or water injection into the clay/molten polymer blend [26-28]. In both cases successful dispersions of the layered silicate were achieved. A similar approach was proposed by Siengchin et al. to obtain toughened POM nanocomposites [29].

Besides, polyethers are reported to favor the untreated clay layers intercalation. Polyether/clay interactions have been studied for years [30]. Particularly, polyethylene oxide (PEO) is even used to quantify the clay surface area [31] as its adsorption is fast and precisely quantified. Concerning general polyethers and clay, the mechanism involves hydrogen bonds between the ether oxygen Lewis base of the polymer chain and the isolated silanols $(\mathrm{SiOH})$ which are strong Brönsted acid sites on the silicate surface [32]. A mechanism involving interactions between hydrophobic siloxanes $(\mathrm{Si}-\mathrm{O}-\mathrm{Si})$ and $\left(\mathrm{CH}_{2}\right)_{\mathrm{n}}-$ segments along polyether backbone [33] has also been suggested. Interlayer's ions are mentioned in the mechanism because of their ability to be complexed by polyethers which then adopt a crown-ether conformation, forming cryptates due to strong $\mathrm{Na}^{+}-$ ether coordinations [34]. Even though the exact mechanism is still in debate, it is clear that there are driving forces for polyethers to enter layered pristine silicates galleries. Indeed, various polyethers were used to successfully prepare nanocomposites filled with pristine montmorillonite (NaMMT) [35, 36] and the efficiency of PEO as intercalant for the preparation of apolar polymers/ NaMMT has been demonstrated [37, 38].

Because of the above mentioned affinities for montmorillonite offered by polyethers and polyamides, trials have been successfully undertaken to prepare polyether-block-amide (PEBA)/clay nanocomposites, varying polyamide and polyether blocks [3941]. However, only organically modified clay has been used and compounding of PEBA/pristine clay nanocomposites has never been reported so far to our knowledge.

Therefore, the present study aims at preparing nanocomposites based on a polyether-block-amide (PEBA) matrix and pristine montmorillonite (NaMMT) by melt-blending with water injection during extrusion as processing aid. Nanostructures are studied by Xray diffraction (XRD) and transmission electron microscopy (TEM). Platelets dispersion is also evaluated by dynamic rheology. Mechanical and thermomechanical properties are studied as well as clay dispersion - material property relationship. Influence of water onto the clay dispersion and onto the polymer structure is evaluated. Organically modified clay and raw unpurified clay are used for a comparison purpose.

\section{Experimental}

\subsection{Materials}

The polyether-block-amide (PEBA) used was a PA12-block-Polytetramethyleneglycol (PTMG) (Pebax 7033 SA 01, Arkema, France). Its molar 
composition is 24.8\% PTMG, $73.0 \%$ PA12 and $2.2 \%$ adipic acid as a linkage [42]. Three kinds of clay were used: an organically modified montmorillonite (Cloisite 30B, Southern Clay Products, USA) further referred to as OMMT, a purified one (Cloisite $\mathrm{Na}^{+}$, Southern Clay Products, USA), referred to as NaMMT and a raw one referred to as RawMMT (Süd Chemie, Germany). RawMMT is a non-purified bentonite, mainly made of NaMMT but also containing non smectic by-products such as quartz, mica, feldspar etc. [43]. Contrary to pristine NaMMT which only contains smectic clay after chemical treatment, the latter silicate is not washed. 'Pristine' refers to NaMMT, whose purification step from bentonite includes the use of surfactants [private communication, Süd Chemie] but is not considered as a modification. Water from lab tap was used during materials preparation. Materials were used as received.

\subsection{Polymer - water solubility characterization}

A high pressure differential scanning calorimeter (Mettler Toledo HPDSC 827 ${ }^{\mathrm{e}}$, Zurich, Switzerland, maximum pressure: 100 bar) was used in order to study the phase separation or miscibility of PEBA and water at high pressure and high temperature. Its measuring chamber is connected to a pressure controlling valve (Brooks P.C. 5866 series) regulated by a Brooks valve controller (ReadOut \& Control Electronics 0152). Pressure and temperature can be independently set in the DSC oven and the heating/cooling curves measured at constant pressure, allowing the simulation of the processing conditions. In order to reproduce the conditions within the extruder, PEBA powder and water were blended at a weight ratio of 70/30, the full sample weighing around $10 \mathrm{mg}$. PEBA pellets were freeze-ground in a grinding device (Pulverisette14, Fritsch/IdarOberstein, Germany/) at $14000 \mathrm{rpm}$.

\subsection{Nanocomposites processing and molding conditions}

PEBA was used as received (vacuum packed) and clays were not dried before extrusion (stored at room conditions: $25^{\circ} \mathrm{C}$, relative humidity $50 \%$ ). Nanocomposites were prepared in a corotating twin screw extruder (Coperion Megacompounder length $=$
Table 1. Composition of the nanocomposites

\begin{tabular}{|l|l|l|c|}
\hline & Clay & Water & $\begin{array}{c}\text { Inorganic content } \\
\text { checked by TGA [wt\%] }\end{array}$ \\
\hline \multirow{2}{*}{$\begin{array}{l}\text { unprocessed } \\
\text { PEBA }\end{array}$} & - & - & 0 \\
\hline \multirow{2}{*}{$\begin{array}{l}\text { extruded } \\
\text { PEBA }\end{array}$} & - & No & 0 \\
\hline \multirow{4}{*}{$\begin{array}{l}\text { PEBA }+ \\
\text { pristine clay }\end{array}$} & - & Yes & 0 \\
\cline { 2 - 4 } & NaMMT & Yes & 2.3 \\
\cline { 2 - 4 } & NaMMT & Yes & 3.9 \\
\cline { 2 - 4 } & NaMMT & No & 4.1 \\
\cline { 2 - 4 } & NaMMT & Yes & 6.4 \\
\hline \multirow{2}{*}{$\begin{array}{l}\text { PEBA }+ \\
\text { modified clay }\end{array}$} & YMMT & Yes & 8.9 \\
\cline { 2 - 4 } $\begin{array}{l}\text { PEBA }+ \\
\text { raw clay }\end{array}$ & OMMT & No & 5.4 \\
\cline { 2 - 4 } & RawMMT & Yes & 4.5 \\
\hline
\end{tabular}

$1000 \mathrm{~mm}, L / D=40$, screw diam: $25 \mathrm{~mm}$, Coperion, Stuttgart, Germany) equipped with a water injection pump and two degassing apertures [44]. The screws are designed so that the melt pressure (70100 bar) at the water injection point stays higher than water vapor pressure curve. Processing temperature was set at $190^{\circ} \mathrm{C}$ all along the screw (actual barrel temperature was $20^{\circ} \mathrm{C}$ lower than the setting value at the water injection point) and rotation speed was $200 \mathrm{rpm}$. Material throughput (polymer + clay) was set at $7 \mathrm{~kg} / \mathrm{h}$ and water input at $3 \mathrm{l} / \mathrm{h}$.

Preliminary thermal analysis of the modified clay indicated that organomodifiers stand for $30 \mathrm{wt} \%$ of OMMT, confirming values reported in the literature [45]. Therefore this organic extra content was taken into account for the final inorganic content to be comparable with other clays. As later displayed (see $\S 4$, Table 1), clays were proportioned so that inorganic contents varied from 0 to $10 \mathrm{wt} \%$ for NaMMT, and set at $5 \mathrm{wt} \%$ for OMMT and RawMMT. Material temperature was measured at the die: 190$195^{\circ} \mathrm{C}$ when water is injected in the melt and 210 $215^{\circ} \mathrm{C}$ for the dry process. Extruded materials were cooled into a water bath and pelletized. The extruded nanocomposites were then vacuum-dried $\left(90^{\circ} \mathrm{C}\right.$, $16 \mathrm{~h}$ ) and injection-molded using an electric machine (Krauss Maffei 80-160E, Munich, Germany) as dumbbell tensile samples according to standard ISO 527-2 (sample type 1A). Injection-molding temperatures were set from $190^{\circ} \mathrm{C}$ (feeding zone) to $230^{\circ} \mathrm{C}$ (nozzle), mold temperature at $20^{\circ} \mathrm{C}$, backpressure at 75 bar; injection screw speed was $80 \mathrm{~mm} / \mathrm{s}$ and holding pressure was kept at 400 bar for $29 \mathrm{~s}$. 


\subsection{Samples structure and properties characterization}

Inorganic content was checked by thermogravimetric analysis (TGA, Mettler Toledo TGA/SDTA851e Zurich, Switzerland) under nitrogen (to prevent the formation of oxidized species that could alter the final weight) from 200 to $500^{\circ} \mathrm{C}$ with a heating rate of $5^{\circ} \mathrm{C}$ per minute, with a stabilization period of 2 minutes at $200^{\circ} \mathrm{C}$. Samples weighing from 7 to $10 \mathrm{mg}$ were die-cut in $1 \mathrm{~mm}$-thick hot-pressed films so as to keep constant dimensions and contact surface.

Molecular weight of the unloaded materials was evaluated by gel permeation chromatography (GPC, Waters Alliance 2695, Waters, Milford, MA, USA) using hexafluoroisopropanol as a solvent at $40^{\circ} \mathrm{C}$. Samples were dissolved during $24 \mathrm{~h}$ at the concentration of $1 \mathrm{~g} / \mathrm{l}$. UV refractometer detector was set at $228 \mathrm{~nm}$ and calibration was performed with polymethyl methacrylate standards. Molecular weights (number average mass $M_{\mathrm{n}}$ and weight average mass $\left.M_{\mathrm{w}}\right)$ are thus reported in 'PMMA-equivalents'.

Structure of the nanocomposites was evaluated using three complementary techniques; transmission electron microscopy (TEM) enables to check the local dispersion and possible exfoliation of the fillers; X-ray diffraction (XRD) analysis quantifies the intercalation in the bulk by indicating the platelets interlayer distance; and rotational dynamic rheology gives an indication of MMT dispersion in the bulk. TEM pictures were taken on the extruded pellets and on injection-molded samples. Ultrathin sections were cut at ambient temperature with a microtome (Leica Reichert FCS) and collected on a 300 mesh copper grid and observed with a TEM microscope (Leo 922 TEM).

The XRD experimental setup was a 2-circles goniometer (Siemens D5000) of $30 \mathrm{~cm}$ radius and $0.002^{\circ}$ positioning accuracy. The incident beam $(\mathrm{Cu}$ $\mathrm{K}_{\alpha}$ radiation, $\lambda=0.15418 \mathrm{~nm}$ ) was obtained from a Rigaku rotating anode operated at $40 \mathrm{kV}$ and $300 \mathrm{~mA}$, fitted with a graphite secondary monochromator and a scintillation counter. Measurements were held on injection-molded samples.

Rheological analyses were performed using an advanced rheometrics expansion system (ARES, Rheometric Scientific, USA) rotational rheometer. As a block copolymer, PEBA keeps some crystalline structure till its order-disorder transition temperature which is reported to be $184^{\circ} \mathrm{C}$ [46]. The experiments were then held at $200^{\circ} \mathrm{C}$ to avoid any ordered state of the matrix and parallel plates were used ( $25 \mathrm{~mm}$ diameter, $2 \mathrm{~mm}$ gap). Linear domains of the different materials were identified from strain sweeps and a common strain of $15 \%$ was then selected for the different samples. Frequency sweeps were run from 0.1 to $100 \mathrm{rad} \cdot \mathrm{s}^{-1}$. Measurements were run three times for repeatability, using new dry samples before each analysis. Storage moduli of the molten nanocomposites are sensitive to nanocomposites structural changes [47]; slopes at low frequency (where the response of the nanocomposites are rather dominated by the polymer/filler interactions) were therefore determined. The latter is considered as a semi-quantitative measure of the degree of exfoliation of the mineral filler [48].

Mechanical properties were evaluated in dynamic and static modes. Rectangular samples $(4 \mathrm{~mm} \times 9 \mathrm{~mm})$ were die cut in $1 \mathrm{~mm}$-thick hot-pressed films for dynamic mechanical analysis (DMA, Mettler Toledo DMA/SDTA 861 $\mathrm{e}$, Zurich, Switzerland) in tensile mode. Oscillating frequency was set at $1 \mathrm{~Hz}$, maximum force at $2 \mathrm{~N}$ and maximum displacement at $8 \mu \mathrm{m}$. Samples were dried in a vacuum oven at $80^{\circ} \mathrm{C}$ for $12 \mathrm{~h}$ before testing. Tests were run from $-125^{\circ} \mathrm{C}$ till $180^{\circ} \mathrm{C}$ at a heating rate of $3^{\circ} \mathrm{C}$ per minute.

Tensile tests were performed on a tensile machine (Lloyd LR50K, UK) using a $5 \mathrm{kN}$ force sensor and an extensometer for strain measurement. Tests were conducted according to ISO 527-1 standard at $50 \mathrm{~mm} / \mathrm{s}$ crosshead speed for the yield strength measurement and at $5 \mathrm{~mm} / \mathrm{s}$ for Young's modulus measurement. Samples were stored during 15 days at $23^{\circ} \mathrm{C}, 50 \%$ relative humidity before testing.

\subsection{Analysis of PEBA-water solubility}

Water injection into the melt can be an efficient way to disperse pristine clay into a matrix by extrusion providing that the polymer exhibits a miscibility with water [22-24]. HPDSC results are reported on Figure 1. For the PEBA/water blend studied at atmospheric pressure, two peaks can be detected for the first heat curve. The first and main one occurs just above $100^{\circ} \mathrm{C}$ and corresponds to water evaporation. The second one occurs at $171^{\circ} \mathrm{C}$ and corresponds to the PA12 block melting. This melting temperature 


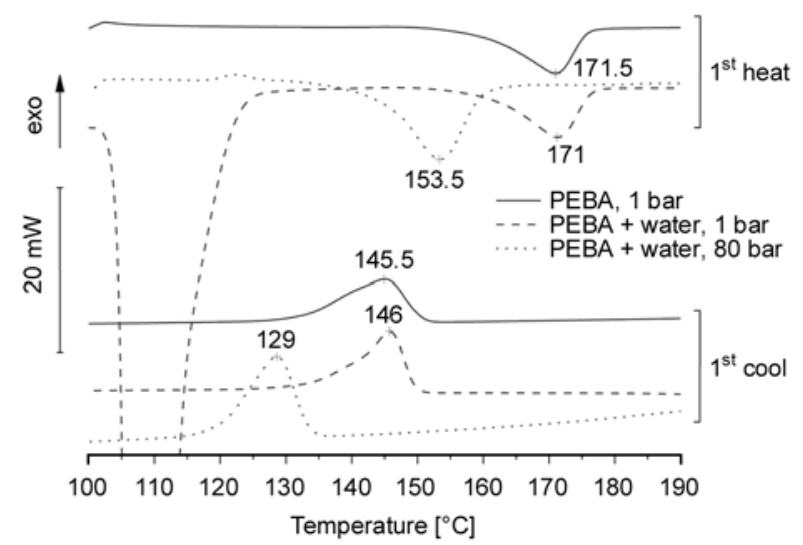

Figure 1. HPDSC of PEBA and PEBA/water blends at $P=$ 1 bar and $P=80$ bar

is not modified compared with pure PEBA since water previously evaporates from the pan. Cooling curves are similar, exhibiting a single crystallization peak at $146^{\circ} \mathrm{C}$ corresponding to PA12 blocks. At high pressure $(80 \mathrm{bar})$ a single peak at $153.5^{\circ} \mathrm{C}$ is observed. As expected, water boiling peak disappeared (at 80 bar pressure, water boiling point is $294.98^{\circ} \mathrm{C}$ [49]) and the remaining peak refers to the PA12 block melting. This peak shifted down by $17.5^{\circ} \mathrm{C}$ due to cryoscopy corresponding to the melting of a single phase made of water and PA12 blocks. In the latter phase, H-bonds make it possible for water to solvate the polyamide blocks [18]. HPDSC measurements were also performed at different pressures $(20,40,60$ and 80 bar, not reported here). Whatever the pressure, the melting point of the PEBA/water phase appears at the same temperature $\left(153.5^{\circ} \mathrm{C}\right)$. This is in good agreement with what has been reported for other polyamides [18, 22-24]. Indeed, the cryoscopic effect played by water onto PA melting is reported when actual pressure stays higher than water vapor pressure curve (for a 20 bar pressure, water boiling point is $214.93^{\circ} \mathrm{C}$ [49]). The cryoscopic effect was also evidenced for the crystallization $\left(17^{\circ} \mathrm{C}\right.$ decrease). The temperature lags were not as high as the ones reported for PA6 $[22,23]\left(\right.$ till $\left.60^{\circ} \mathrm{C}\right)$ on the one hand because the matrix is a copolymer (PA12 blocks are reported to stand for $88 \mathrm{wt} \%$ [46] or for $73 \mathrm{~mol} \%$ [40] of the copolymer) and on the other hand because of the lower H-bonding concentration among PA12 compared with PA6. Nevertheless, the HPDSC results confirm that water and the polyamide part of PEBA behave as a single molten phase in the extrusion conditions.
Consequently, PEBA fits the requirements for its diffusion and adsorption onto the clay surface, according to the mechanism described by Fedullo et al. [44] in the case of PA6/NaMMT nanocomposites. Indeed, the PEBA/water miscibility improves the polymer ability to diffuse into the clay by increasing its polarity and decreasing its viscosity. Then the macromolecules can adsorb on the mineral and concomitantly desorb the $\mathrm{H}_{2} \mathrm{O}$ molecules previously adsorbed on the surface, which would not be possible if PEBA and water were immiscible.

\section{Analysis of PEBA/clay nanocomposites structure}

\subsection{Inorganic content}

Inorganic content of the compounded materials is reported in Table 1. Mineral contents referred to later correspond to the values measured by TGA under nitrogen flow.

\subsection{Molecular mass}

Melt processing of polycondensates, such as polyamides, usually requires cautious water removal from the resin in order to avoid backbone hydrolysis (amide links are broken into carboxy and amine ends). Even low moisture contents lead to hydrolytic chain scission and strong reduction of the molecular mass [50].

In the present work, injected water represents $30 \mathrm{wt} \%$ of the total extrusion throughput. The backbone degradation state was therefore checked by GPC. Table 2 presents molecular masses of the PEBA matrix (in PMMA equivalents) as received and after extrusion with or without water.

A decrease of the molecular mass is observed for PEBA extruded without water injection in comparison with the initial sample $\left(M_{\mathrm{n}}:-7.4 \%\right.$ and $M_{\mathrm{w}}$ : $-11.6 \%)$. This decrease is mainly attributed to the thermal decomposition of the polymer blocks [51]. For PEBA extruded with water, molecular masses are similar to the unprocessed material $\left(M_{\mathrm{n}}:+1.3 \%\right.$ and $M_{\mathrm{w}}$ : $\left.-4.8 \%\right)$. Expected decreases of the chain

Table 2. Number $\left(M_{\mathrm{n}}\right)$ and weight $\left(M_{\mathrm{w}}\right)$ average mass of PEBA before and after extrusion step. Results are given as PMMA equivalents.

\begin{tabular}{|c|c|c|}
\hline & $\mathrm{M}_{\mathrm{n}}\left[\mathrm{g} \cdot \mathrm{mol}^{-1}\right]$ & $\mathbf{M w}\left[\mathrm{g} \cdot \mathrm{mol}^{-1}\right]$ \\
\hline unprocessed PEBA & 39100 & 82800 \\
\hline PEBA extruded with water & 39600 & 78800 \\
\hline PEBA extruded without water & 36200 & 73200 \\
\hline
\end{tabular}


length originating from the thermal decomposition and the hydrolysis are hardly observed when water is injected during extrusion. On the contrary, water prevents the matrix from any thermal degradation in the barrel by plasticizing and lubricating the processing medium. This observation has already been reported even though the mechanism is still unclear [52].

Injecting water during extrusion prevents PEBA from thermal degradation and does not lead to the hydrolysis of the polyamide blocks, due to rather slow kinetics and short residence time (less than 30 seconds in contact with water).

\subsection{Transmission electron microscopy}

TEM pictures of materials prepared with OMMT are reported in Figure 2 at low and high magnification. Dispersion is homogeneous as visible at low magnification and, in addition to the intercalated
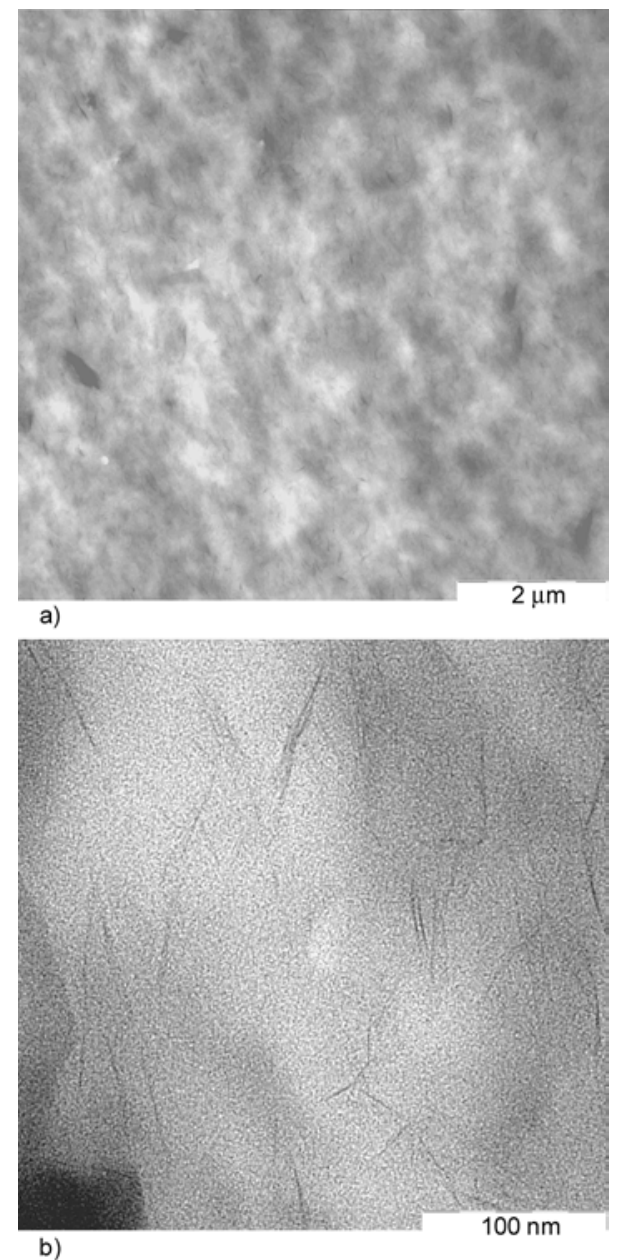

Figure 2. TEM observation of injection-molded $5.5 \mathrm{wt} \%$ OMMT/PEBA nanocomposite prepared without water injection at low (a) and high magnification (b)

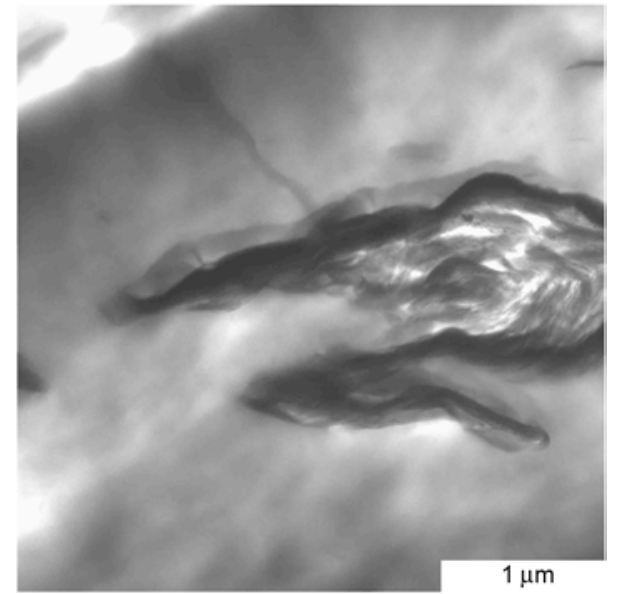

Figure 3. TEM observation of injection-molded $4.1 \mathrm{wt} \%$ NaMMT/PEBA nanocomposite prepared without water injection

layers, some part of the mineral exhibit an exfoliated state at high magnification. Samples prepared with (not shown) and without water present similar structures.

Figures 3 and 4 show TEM images of nanocomposites prepared with 4.1 and $3.9 \mathrm{wt} \%$ pristine clay, respectively without and with water injection. At low magnification, the images reveal the presence of micrometric tactoids for the dry processed materials (Figure 3), while the dispersion is almost homogeneous for PEBANaMMT prepared with water injection (Figure 4). This composite exhibits tactoids of reduced dimensions and single exfoliated layers are detectable at high magnification.

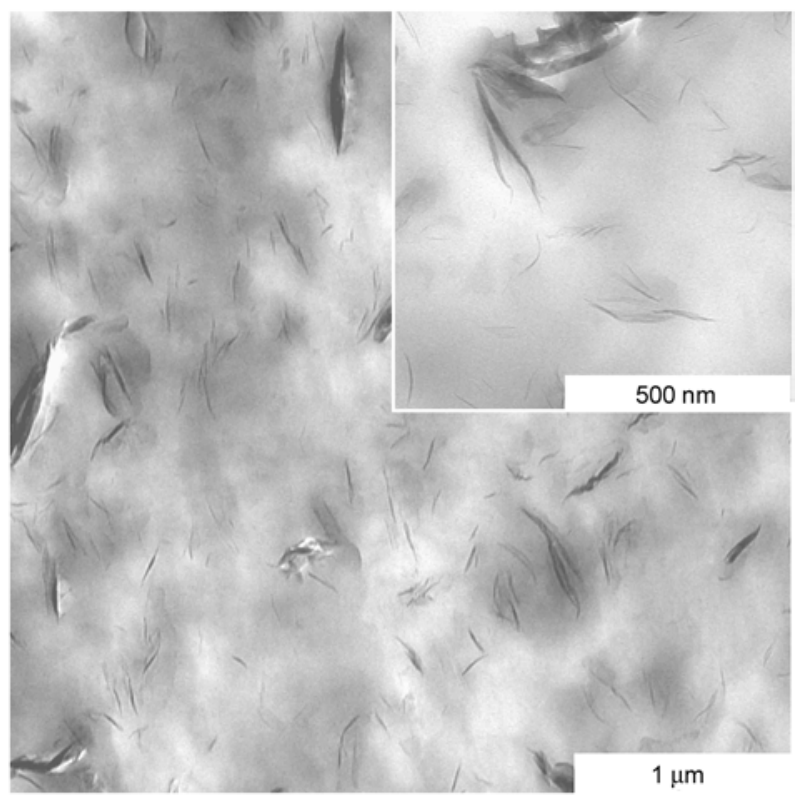

Figure 4. TEM observations of injection-molded $3.9 \mathrm{wt} \%$ NaMMT/PEBA nanocomposites prepared with water injection 


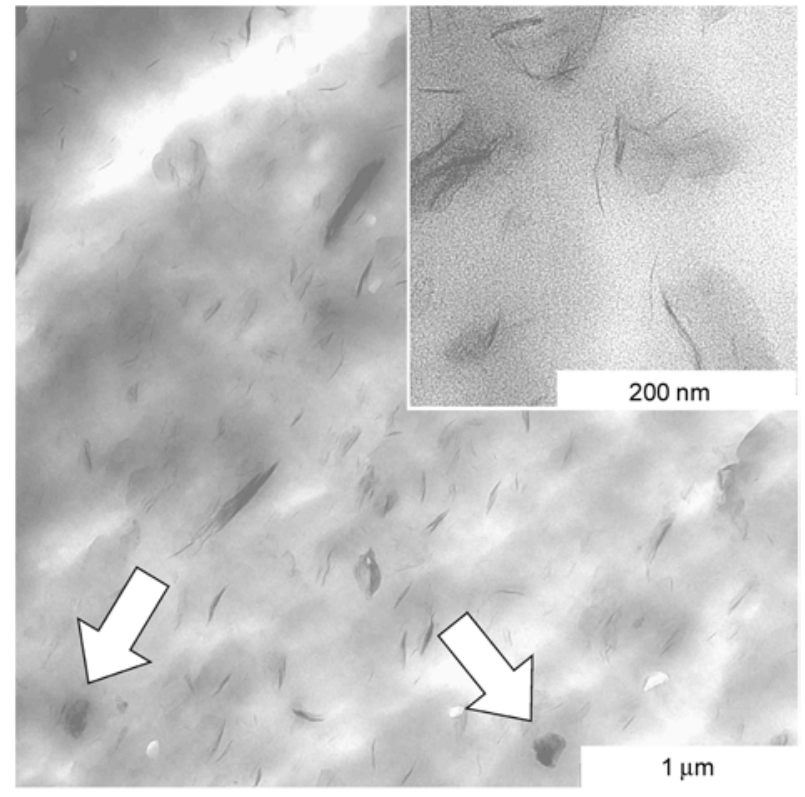

Figure 5. TEM observation of $4.7 \mathrm{wt} \%$ RawMMT/ PEBA nanocomposites (extruded pellet) prepared with water injection. Non smectic minerals are indicated by arrows.

The low magnification TEM pictures of the materials prepared with RawMMT when water is injected during extrusion show a structure (Figure 5) close to the one of PEBA/NaMMT with water. Moreover, the presence of black spots which are non smectic minerals (quartz etc.) is also visible in Figure 5 as indicated by arrows. Intercalated and exfoliated layers can be detected at high magnification. Samples prepared with RawMMT without water injection exhibit a structure close to that of PEBA/ NaMMT extruded in the same conditions.

According to these microscopy observations, water actually improves NaMMT and RawMMT dispersions in the PEBA matrix, both for the homogeneity and the exfoliation of the mineral, even if the materials prepared with OMMT exhibit a better dispersion degree. On the basis of TEM observations, water injection has only a limited effect, if any, on the PEBA/OMMT nanocomposites.

\subsection{X-Ray diffraction}

Intercalation of the mineral by the PEBA chains can be quantified by X-ray diffraction (XRD) measurement. It provides the interlayer distance (corresponding to $d_{001}$, distance between two consecutives clay layers) according to Bragg's law. Figure 6 shows XRD patterns corresponding to the nanocomposites constitutive components in the $2 \theta$ range:

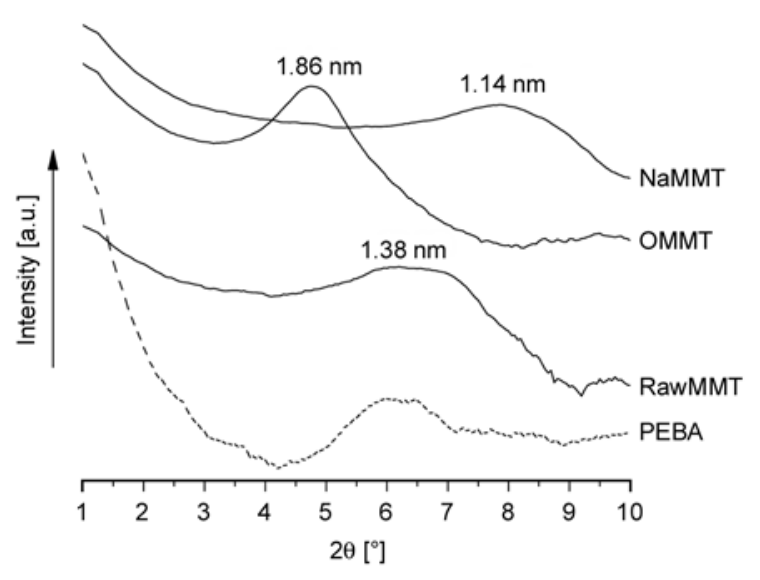

Figure 6. XRD patterns of the constitutive components

[1-10 ${ }^{\circ}$. OMMT exhibits a peak corresponding to an interlayer spacing of $1.86 \mathrm{~nm}$ which is higher than that of NaMMT and RawMMT (1.14 and $1.38 \mathrm{~nm}$ respectively). This result is confirmed in the literature $[3,5]$ and explained by the fact that minerals are modified by exchanging initial sodium ions with bigger alkylammoniums in order to change the clay polarity and pre-exfoliate the layers. The difference between the two non-modified clays may be explained by the different origins of the minerals and by the moisture content (due to the structural difference of RawMMT). For instance, the basal spacing is known to highly depend on the moisture content of the mineral (the higher the latter, the more distant the platelets) [53] and on the counterion type [12].

PEBA exhibits a diffraction peak around $6^{\circ}$, which corresponds to a crystalline phase of the PA12 block (corresponding to the (200) plane of pseudohexagonal $\gamma$-form crystal whose reflection peak should appear at $2 \theta=5.5^{\circ}$ [54]). This may complicate the interpretation of the XRD patterns of the nanocomposites, as this peak position is precisely in the angle range where clay $d_{001}$ peaks may shift. The XRD patterns of the nanocomposites containing OMMT (Figure 7) exhibit two peaks located at $5.5^{\circ}$ and around $2.5^{\circ}$, whatever the processing conditions (with or without water). The former peak refers to PEBA diffraction and the latter to the mineral $d_{001}$ peak. The corresponding basal distance (around 3.5-4.0 nm) is increased compared with the neat OMMT $(1.86 \mathrm{~nm})$ indicating an intercalation by the polymer. Both nanocomposites present identical interlayer distances. Consequently, water injection during extrusion does not seem to have any 


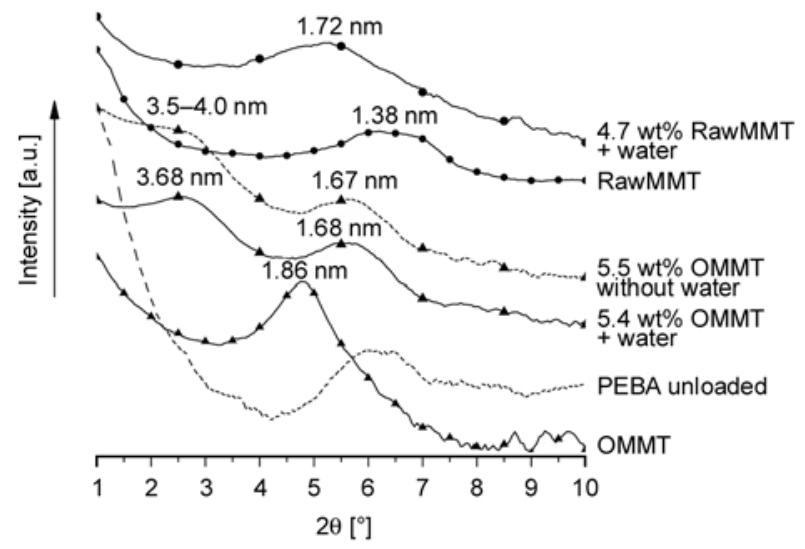

Figure 7. XRD patterns of PEBA/OMMT nanocomposites

effect in the case of PEBA/OMMT nanocomposites, as far as intercalation is concerned.

XRD patterns of PEBA/NaMMT nanocomposites are shown on Figure 8. In the case of materials prepared with water injection, XRD patterns are almost identical whatever the clay content. The neat NaMMT peak located at $2 \theta=7.7^{\circ}$ is no more visible. Curves present a single broad peak around $5.4^{\circ}\left(d_{001}=\right.$ $1.65 \mathrm{~nm}$ ) which is analogous to the diffraction peak exhibited by pure PEBA. However, no other peak is detected for PEBA/NaMMT compositions and TEM observations did not allow to consider that clay layers are fully exfoliated. Therefore, the peak located around $5.4^{\circ}$ should be assigned to a combination of the matrix diffraction peak and the clay $d_{001}$ peak which has shifted towards lower angles.

The nanocomposite prepared with $4.1 \mathrm{wt} \%$ NaMMT without any water injection exhibits two diffraction peaks $\left(5.4^{\circ}\right.$ and around $\left.9^{\circ}\right)$. The first one corresponds to the matrix as detailed previously and the second one may be ascribed to the clay $d_{001}$ diffraction peak. The corresponding interlayer distance

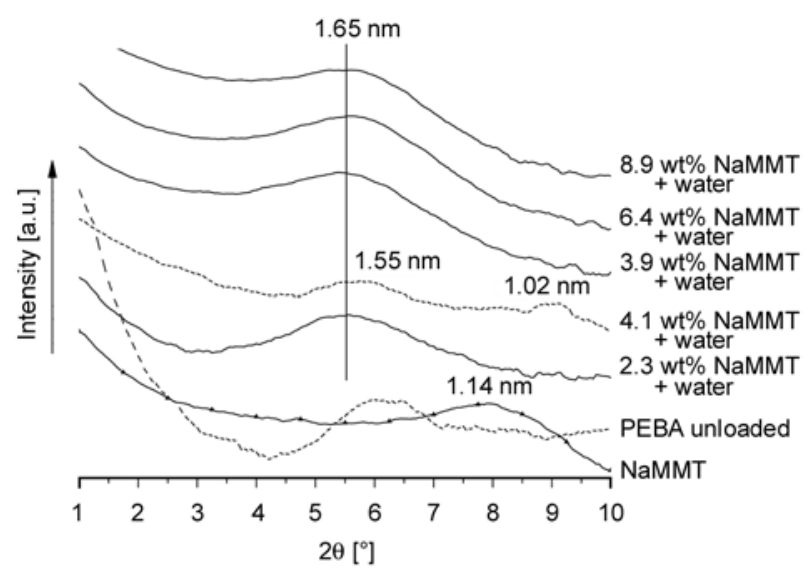

Figure 8. XRD patterns of PEBA/NaMMT nanocomposites
$(1.02 \mathrm{~nm})$ is lower than the neat clay (interlayer distance: $1.14 \mathrm{~nm}$ ) and this reduction can be explained by a drying of the clay during extrusion procedure. For PEBA/RawMMT nanocomposites, clay is intercalated and basal spacing increased by $0.34 \mathrm{~nm}$ in case of water injection (results not reported here), while platelets are compacted by $0.35 \mathrm{~nm}$ compared with the neat RawMMT when no water is injected. These compactions can be explained by the fact that neat clays were analyzed by XRD without being previously dried. During the extrusion process without water injection, the added mineral undergoes an in situ drying step; initial water molecules are removed from the clay surface and $d_{001}$ decreases. According to XRD analysis water injection leads to the intercalation of NaMMT and RawMMT by PEBA (otherwise clays should remain in a tactoid state), whereas it hardly modifies the intercalation state of OMMT.

\subsection{Rheology}

Rheology is one of the easiest and best way to quantify the filler dispersion into polymer nanocomposites, loaded with carbon nanotubes $[55,56]$ or with clay platelets [57]. Effects are more noticeable on the storage modulus $\left(G^{\prime}\right)$ than on the loss modulus. In particular slopes and absolute values of $G^{\prime}$ at low frequencies are indicators of the dispersion state of the nanocomposite $[47,58]$. In the high frequency range, the signal stands for the segmental motion of the macromolecules, which is directly linked to the molecular mass.

Storage moduli versus rotational frequency are reported in Figure 9. The Figure 9a presents curves corresponding to materials prepared with water injection and the corresponding ones prepared without. For pure PEBA, water injection increases $G^{\prime}$ at high frequency (from $1900 \mathrm{~Pa}$ to $6900 \mathrm{~Pa}$ at $19.3 \mathrm{rad} \cdot \mathrm{s}^{-1}$ ). This $G^{\prime}$ improvement at high frequency is noticeable for loaded PEBA/clay nanocomposites as well. According to the previously presented GPC results, this improvement can be attributed to a difference in molecular weights. The phenomenon of lower degradation due to the use of water, already evidenced for the neat (unfilled) PEBA, also occurs for the PEBA matrix of the various nanocomposites.

The Figure 9b shows storage moduli of the PEBA/ clay nanocomposites of various clay contents. $G^{\prime}$ 

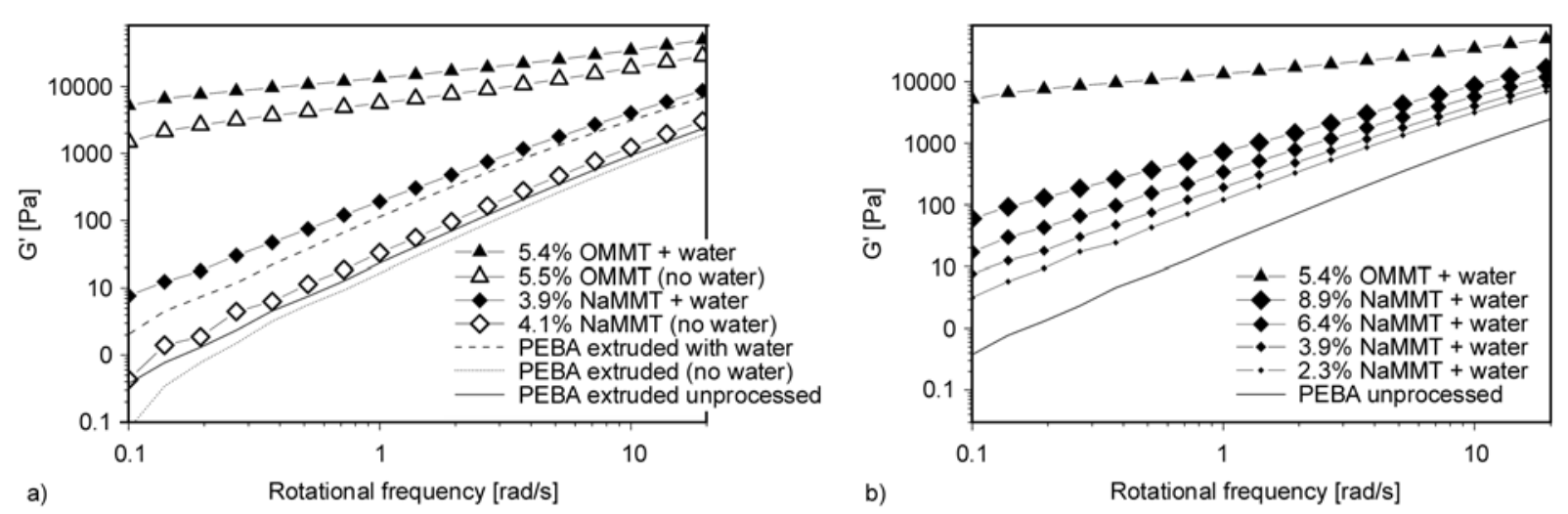

Figure 9. Storage modulus of PEBA/Clay nanocomposites with/without water injection (a) and for different clay contents (b)

Table 3. $G^{\prime}$ slopes at low rotational frequencies for PEBA/ clay nanocomposites

\begin{tabular}{|l|c|}
\hline & $\begin{array}{c}\boldsymbol{G}^{\prime} \text { slope at low } \\
\text { rotational frequency }\end{array}$ \\
\hline PEBA non extruded & 1.81 \\
\hline PEBA + 2,3\% NaMMT + water & 1.56 \\
\hline PEBA + 3,9\% NaMMT + water & 1.38 \\
\hline PEBA + 4,1\% NaMMT without water & 1.89 \\
\hline PEBA + 6,4\% NaMMT + water & 1.31 \\
\hline PEBA + 8,9\% NaMMT + water & 1.09 \\
\hline PEBA + 5,4\% OMMT + water & 0.42 \\
\hline PEBA + 5,5\% OMMT without water & 0.61 \\
\hline
\end{tabular}

slopes at low frequency are presented in Table 3 (calculated using an exponential fitting between 0.1 and $0.5 \mathrm{rad} \cdot \mathrm{s}^{-1}$ ). For PEBA/NaMMT nanocomposites the storage modulus is all the higher as the mineral content increases and differences between curves expand at low frequencies. Moreover, slopes at low frequency decrease concomitantly. For the neat PEBA, the slope is equal to 1.81 which deviates slightly from the normal terminal slope for homopolymer melts (a value of 2 is theoretically expected). When $8.9 \mathrm{wt} \%$ NaMMT is added to PEBA, this value falls down to 1.09 . Such a $G^{\prime}$ increase is frequently reported and has been detailed as $G^{\prime}=G_{\text {matrix }}^{\prime}+G_{\text {confine }}^{\prime}+G_{\text {inter }}^{\prime}[59,60]$. The latter part comes from filler-filler frictional interactions and $G_{\text {confine stands for macromolecules }}^{\prime}$ intercalated in between clay layers. $G^{\prime}$ inter mainly contributes to the increase at low frequency of $G^{\prime}{ }_{\text {nano, }}$, particularly when clay content is above the percolation threshold [60-62]. Similar results were reported in the literature for PA12/NaMMT prepared by solvent intercalation. $G^{\prime}$ slope at low frequency was 1.6 for the neat polymer and lowered to
1.4 and 1.2 for 2 and $4 \mathrm{wt} \%$ NaMMT loading, respectively [58].

For the two PEBA/NaMMT samples prepared at similar mineral contents (3.9 and $4.1 \mathrm{wt} \%$ NaMMT with and without water injection respectively) the modulus at low frequency increases drastically (more than one decade) and slope decreases (from 1.89 to 1.38) when the dispersion shifts from tactoid to intercalated/exfoliated (when water is used). Those two materials exhibit deeply different structures as evidenced by XRD and TEM. These results clearly emphasize that $G^{\prime}$ value and its concomitant slope are more sensitive to the quality of the clay dispersion than to the clay content. Clusters are much more numerous in the case of an intercalated and exfoliated nanocomposite than for a microcomposite such as PEBA/NaMMT prepared without water. Macromolecules of restricted mobility are less numerous for this material compared with the corresponding water-processed one.

Values of $G^{\prime}$ obtained with modified clays nanocomposites are the highest and even higher when water is used. The slope is 0.42 for PEBA $/ 5.4 \mathrm{wt} \%$ OMMT nanocomposite. This plateau reached by $G^{\prime}$ at low frequency for PEBA/OMMT nanocomposites is the typical rheological signature of a high clay dispersion [63]. In the case of PEBA/OMMT prepared with or without water, clay platelets and tethered macromolecules have formed a three-dimensional superstructure. Clusters are uniformly dispersed all among the bulk. The slight difference in rheological response induced by water injection probably comes from the matrix degradation, which is limited when water is injected. Nevertheless, the clay dispersion 
states are better than in the case of PEBA/NaMMT nanocomposites.

Results obtained by rheological analysis confirm and supplement the structural analysis obtained by $\mathrm{XRD}$ and TEM. The water injection reduces PEBA degradation during extrusion and is required in order to get NaMMT dispersion (close to OMMT), intercalation and partial exfoliation.

\section{Analysis of PEBA/clay nanocomposites properties}

In order to establish the nanostructure-property relationship of the PEBA/clay nanocomposites, mechanical properties were measured.

\subsection{Dynamic mechanical properties}

Figure 10a shows storage moduli (relative to PEBA, which was measured at $707 \mathrm{MPa}$ ) at $25^{\circ} \mathrm{C}$ of the prepared nanocomposites versus mineral content. The relative modulus increases linearly as a function of the NaMMT content when water is used. For a given NaMMT content, water injection improves the material modulus by $28 \%$. The modulus of PEBA/NaMMT prepared without water is close to the one of pure PEBA. Such an improvement of the storage modulus either by increasing the clay content or by improving the dispersion state using water injection is directly linked to the improved interactions between the matrix and the filler, and not only to the volume fraction of the high modulus clay [2].

Nanocomposites prepared with modified clay (OMMT) exhibit a modulus slightly higher than the one filled with NaMMT. This is probably due to the better dispersion state and subsequent larger polymer/platelets interphase. When water is used, the corresponding PEBA/organoclay nanocomposite

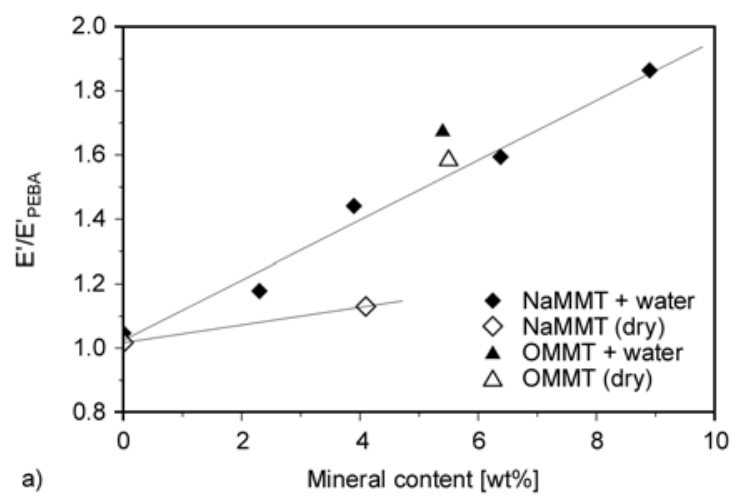

exhibits a slightly higher $E^{\prime}$ compared with the one prepared without water. This modulus difference may be inherent to the composition of the matrices as the dispersion states are similar.

It is also worth noting that the results obtained with PEBA/NaMMT are even better than the ones reported in the literature for PA6 filled with OMMT. Indeed, at $5 \mathrm{wt} \%$ clay loading, the normalized $E^{\prime}$ is 1.33 [2] or 1.34 [64] while it has been found to be 1.44 in the present study.

Figure 10b shows the glass transition temperature $\left(T_{\mathrm{g}}\right)$ of the PEBA matrix versus mineral content, which corresponds to PA12 blocks. Indeed, glass transition corresponding to PTMG blocks is hardly detectable because, on the one hand the soft block content is low, and on the other hand a PA12 $\beta$-transition appears in this temperature range [65]. In the case of NaMMT clay, $T_{\mathrm{g}}$ increases when the mineral content increases, from 29 to $35^{\circ} \mathrm{C}$. The $T_{\mathrm{g}}$ of the PEBA $/ 4,1 \mathrm{wt} \%$ NaMMT compound prepared without water injection is the same as the pure PEBA matrix. When water is injected, the $T_{\mathrm{g}}$ increases by $3^{\circ} \mathrm{C}$ (PEBA $+3.9 \mathrm{wt} \%$ NaMMT + water). This increment can be assigned to a reduced mobility of the macromolecular chains adsorbed on the mineral platelets [66]. The more exfoliated the mineral is, the less mobile the chains and consequently the higher the $T_{\mathrm{g}}$ are.

In the case of PEBA/OMMT nanocomposites prepared with or without water, $T_{\mathrm{g}}$ is increased compared with the pure polymer. It must be considered that for these modified-clay compounds, the macromolecules mobility is highly reduced by the homogeneous platelets dispersion. Such a dispersion leads to high $T_{\mathrm{g}}$ in spite of an eventual plasticizing effect provided by alkylammoniums. Indeed, some authors reported that $T_{\mathrm{g}}$ of PA12/OMMT nanocomposites is

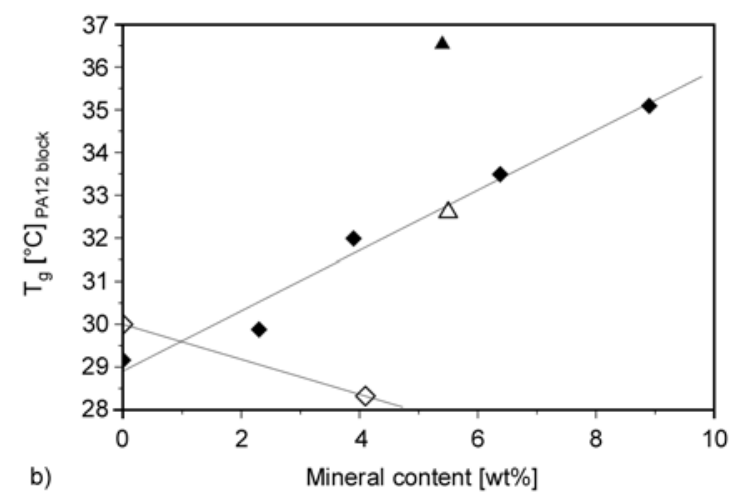

Figure 10. Relative storage modulus (a) and glass transition temperatures (b) of PA12 blocks of PEBA/Clay nanocomposites measured by DMA in tensile mode 
independent of the clay content [5] because the mobility restriction is notably counterbalanced by the water plasticization. Moreover, a decrease of the nanocomposite glass transition, justified by a plasticizing effect attributed to the alkylammonium ions modifying the clay, is also reported when the mineral content increases [3]. Besides, mechanism of polyamide nanocomposites plasticization is the same as for the unfilled polyamide [66, 67]. PEBA/OMMT nanocomposite prepared with water injection exhibits a $T_{\mathrm{g}}$ higher than that of the one prepared without water. In combination with the slight structural dissimilarity detected by rheology, a plasticization phenomenon may explain this neat glass transition difference. The water-prepared PEBA/OMMT nanocomposites are less plasticized by alkylammonium because of a steam stripping of the surfactant moieties throughout the degassing apertures. Detailed results of odours and volatile organic compounds (VOC) emission analyses performed on the processed materials are reported elsewhere [67]. One of the key points is that PEBA/ OMMT prepared without water injection emits much more odours and VOCs (coming from the alkylammonium or its decomposition products) than the corresponding nanocomposite prepared with water injection. The latter emits as few VOCs as the neat PEBA. This result confirms the flushing of the plasticizing surfactant from the OMMT-filled nanocomposite when water is injected [68]. A similar phenomenon is reported in the literature for polymer/cellulose composites prepared with water injection into the melt. Cellulose degradation products responsible for the coloration of the material were steam-extracted and resulting material exhibited a limited coloration [52]. Finally, it is worth noticing that materials exhibiting

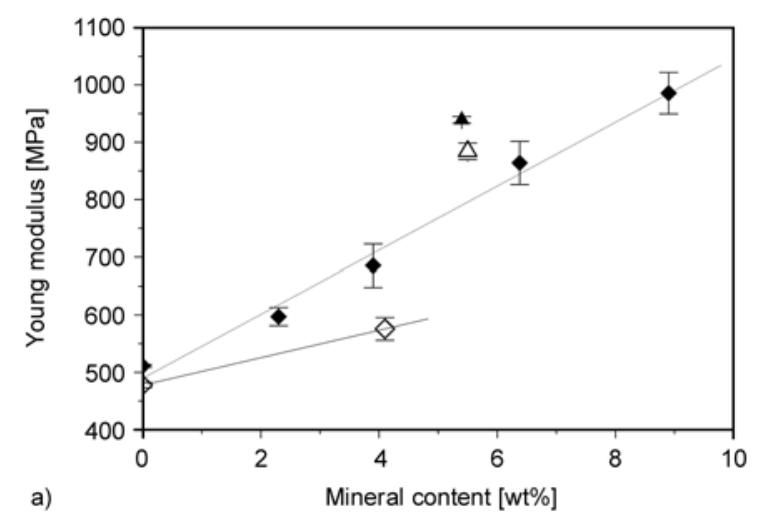

similar structures detected by XRD and TEM (and limited differences in rheological responses) present different thermomechanical behaviors because of slight changes in the chemical composition (organo-modifiers account for less than $2 \mathrm{wt} \%$ of the PEBA/OMMT nanocomposites).

At last, the glass transition increment $\left(\Delta T_{\mathrm{g}}\right)$ presented by the material containing $5.5 \mathrm{wt} \%$ OMMT prepared without water is comparable with the one measured for water-prepared and NaMMT-filled nanocomposite at similar (4.1 wt \%) clay loading. When water is injected, PEBA/OMMT exhibits the highest improvement of glass transition $\left(+7^{\circ} \mathrm{C}\right)$ of the series. The organomodifications and use of water enabled the fine dispersion of the clay layers and water clears the nanocomposite from the chemicals coming from the clay grafting treatment. The role played by water is different in the case of PEBA/NaMMT nanocomposites. Water allows the intercalation of pristine platelets by macromolecules whose mobility is then reduced as evidenced by $T_{\mathrm{g}}$ measurements.

\subsection{Tensile properties}

Results of tensile tests are reported in Figure 11. Figure 11a shows the Young modulus, defined as and Figure $11 \mathrm{~b}$ the yield stress. Young modulus is a measure of the stiffness and is the tangent modulus of the initial, linear portion of a stress-strain curve. Yield stress is defined as the stress that can undergo the material before plastic flow. Modulus of neat PEBA extruded with water injection is slightly higher $(+7 \%)$ than that of PEBA extruded without water, probably because of a limited degradation as evidenced previously by GPC and rheological analysis. The higher modulus values observed for nano-

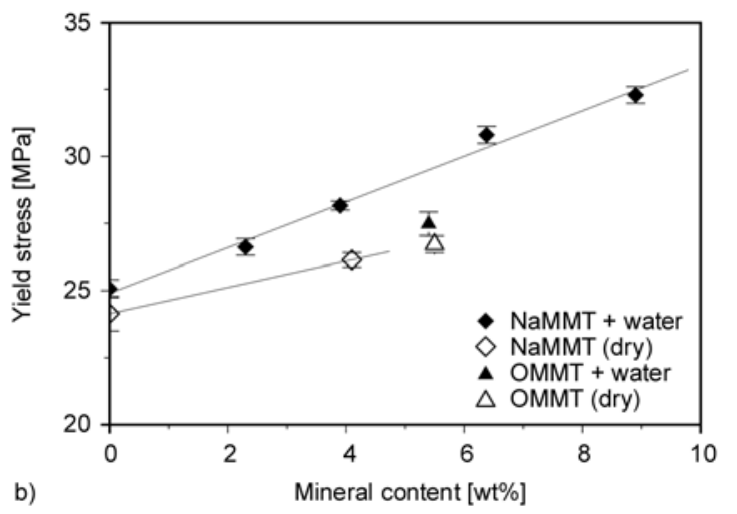

Figure 11. Tensile mechanical properties of PEBA/Clay nanocomposites (a: Young modulus; b: Yield stress). Error bars represent the standard deviation. 
composites prepared with water injection also result from the higher matrix molecular weights, as a minor contribution.

In the case of NaMMT-filled materials prepared with water injection, Young modulus increases linearly with the mineral content; it is almost doubled at $8.9 \mathrm{wt} \%$ clay loading. However, for a given NaMMT content (around $4 \mathrm{wt} \%$ ), the modulus is decreased by $16 \%$ if water is not injected during extrusion and is close to the unloaded PEBA modulus. As demonstrated previously the dispersion remains at 'intercalated and partially exfoliated' state for all PEBA/ NaMMT nanocomposites prepared with water injection. Consequently the volume of polymer/clay interphase increases along with NaMMT content. The interphase is not only made of the adsorbed chains but also includes macromolecules entangled with the former ones. A characteristic structure of limited conformation is then created at the vicinity of the filler. Mechanical properties of this interphase strongly differ from the bulk polymer [67]. Increasing the NaMMT content improves the interphase volume and its contribution to the mechanical behavior of the whole material, as usually reported for intercalated/exfoliated nanocomposites $[2,3,5,70]$. Injected water improves the dispersion state and the volume of polymer/clay interphase increases as evidenced by the structural analysis. The resulting modulus is thus enhanced, although the clay loading is unchanged.

Compared to the materials filled with pristine clay, the OMMT-filled nanocomposites exhibit a slightly higher Young modulus, due to the better clay dispersion. Moreover, improvement of modulus of PEBA/ OMMT owing to water injection is limited $(+6 \%)$. It can be attributed, on the one hand to the higher molecular mass obtained, and on the other hand to the absence of plasticization by alkylammoniums when water is used, as previously discussed for glass transition results.

Finally, as previously highlighted by rheological measurements, the interphase volume among PEBA/ NaMMT nanocomposites increases with the mineral loading and water injection. For PEBA/OMMT nanocomposites, the little difference induced by water injection, previously revealed by rheological analysis, remains detectable when Young modulus is considered. Results obtained in static and dynamic modes are similar and confirm each other.
Figure $11 \mathrm{~b}$ shows the yield stresses of the studied nanocomposites. In the case of nanocomposites filled with NaMMT, when water is injected during the melt processing, the yield stress increases with the pristine mineral content (up to $+30 \%$ for $8.9 \mathrm{wt} \%$ clay). The nanocomposites based on NaMMT prepared without water injection have a lower yield stress. This may be attributed to the lower dispersion and also eased plastic deformation due to the presence of aggregates which induce decohesion leading to microcrazing. Both PEBA/OMMT nanocomposites exhibit slight yield stress improvements, clearly inferior to what is observed with NaMMT. Water injection has little effect onto PEBA/OMMT nanocomposites. In spite of the higher rigidity (high Young modulus) of these nanocomposites and a good dispersion as attested by rheology and TEM, the decrease of the yield stress means that the plastic deformation is eased. One can suppose that the increase of the interface proportion increases the number of crazing sites which in turn help the initiation of plastic deformation.

Elongations at break are reported on Figure 12. PEBA/NaMMT nanocomposites prepared with water injection break at lower elongation rates than neat PEBA. This ductility reduction is amplified when the mineral content increases. When no water is injected during extrusion of PEBA $/ 4 \mathrm{w} \%$ NaMMT compound the elongation at break is higher than when water is injected, and unchanged in comparison with pure PEBA. The corresponding microcomposite exhibits the highest elongation at break of the filled materials' series. This result can be explained by the fact that microcomposites and

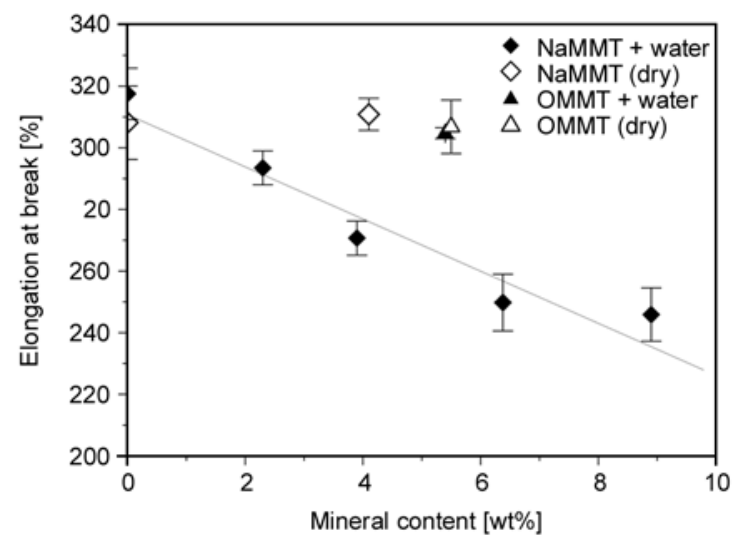

Figure 12. Relative elongation at break of PEBA/clay nanocomposites. Error bars represent the standard deviation. 
nanocomposites undergo different fracture behaviors, as reviewed by Cotterell et al. [71]. According to the authors, semicrystalline polymer nanocomposites are toughened through to two mechanisms: particles' delamination and matrix deformation. For the latter, energy is mainly absorbed because of the formation of multiple craze-like bands while shear yielding is reported to be negligible. Moreover, particles' delamination prevails upon matrix deformation when particles are large [71]. In the case of clay nanoplatelets poorly dispersed into PA12, Kim et al. [72] report that the deformation process is governed by the formation of microvoids inside the filler tactoids, which toughens the material. The mechanism is illustrated in Figure 13. Such a tactoid delamination process may take place in PEBA/ NaMMT prepared without water injection, whose dispersion state is poor, as evidenced previously. On the contrary, for the best dispersed nanocomposites (PEBA/OMMT, with or without water injection), the energy absorbing mechanism cannot be particles' delamination as most of the clay is dispersed as single layers. These exfoliated nanocomposites exhibit higher elongations at break owing to energy absorbing matrix deformation. Crazes are initiated at the vicinity of the platelets. These fibrillated microvoids act as energy dissipaters if the platelets are well dispersed. Otherwise crazes may cause the failure of the material [73]. The homogeneous clay dispersion observed in PEBA/OMMT

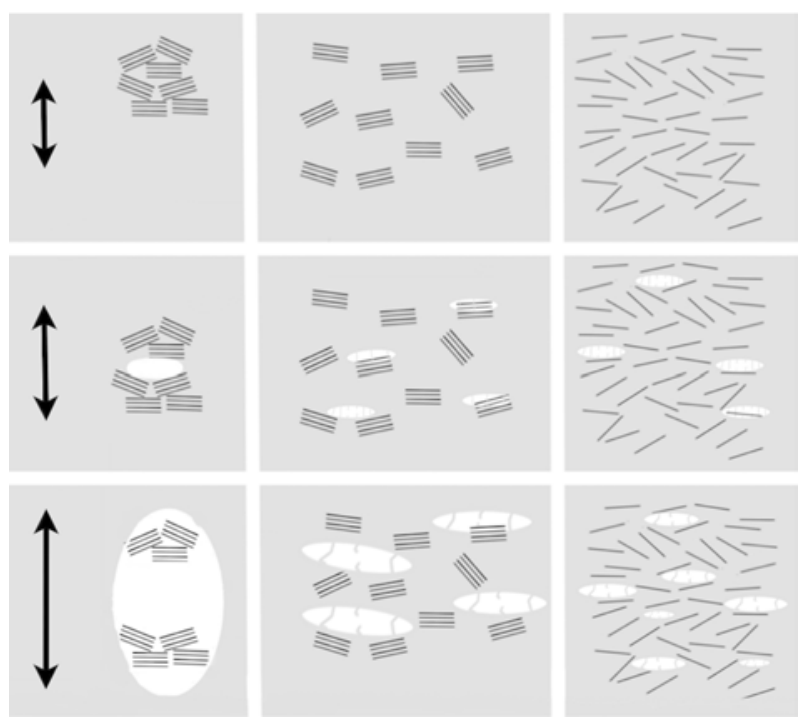

Figure 13. Deformation mechanisms of PEBA/clay microcomposite (left), intercalated nanocomposite (center) and exfoliated nanocomposite (right). Arrows indicate the loading direction and amplitude leads to the uniform creation of crazes among the bulk. Once nucleated, crazes grow and concomitantly disperse the energy provided by the strain (Figure 13). Transformation of those safe and scattered microvoids into disastrous cracks requires a high density of crazes or their coalescence, and consequently appears at high elongation rates [70]. Decohesion of the filler from the matrix is not considered here because of the strong interactions between PEBA and clay platelets in the exfoliated and intercalated structures.

Concerning intercalated nanocomposites, Cotterell et al. [71] report numerous and contradictory interpretations. However, in the case of PEBA/NaMMT nanocomposites prepared with water injection, whose morphologies are mainly intercalated but also exhibit small tactoids, it is clear that the toughening mechanism is hardly influenced by particles' delamination. Moreover, the craze formation does not toughen the materials as for exfoliated nanocomposites, even if the crazes are located close to the layers as well. For intercalated nanocomposites, stress fields created around the crazes may overlap as the platelets are not homogeneously dispersed $[69,72]$. Then, adjacent microvoids may coalesce and favor the development of cracks which lead to the material breakdown as illustrated in Figure 13. Intercalated PEBA/NaMMT hardly dissipate energy under tension because of their structure. On the one hand it does not reveal the presence of large aggregates required for delamination to take place; and on the other hand it is not exfoliated enough for crazes to safely toughen the material. Such an intermediate dispersion state consequently embrittles the material. The embrittlement is all the higher as the clay content increases, acting as crack initiators. Finally, the tensile performances obtained with NaMMT are comparable with the ones reported in the literature [4]. Wilkinson et al. [4] report that tensile modulus and yield stress are improved by $73 \%$ and $28 \%$ respectively when $5 \mathrm{wt} \%$ OMMT is incorporated in a PA6 matrix. For PEBA $+6.4 \mathrm{wt} \%$ NaMMT prepared with water injection, these improvements reach 89 and $30 \%$ respectively. Concerning elongation at break, Wilkinson reports a 93\% decrease whereas the present PEBA/NaMMT nanocomposites exhibits a $43 \%$ decrease only. Moreover, tensile performances reached by PA/ NaMMT prepared with in the same conditions [28] 
are clearly lower than what is reported here for PEBA/NaMMT.

\section{Conclusion}

The aim of the present study was to elaborate nanocomposites based on a polyether-block-amide (PEBA) matrix and pristine montmorillonite using water injection during extrusion as processing aid. PEBA nanocomposites based on organo-modified montmorillonite were used as a reference. High pressure differential calorimetry evidenced that the miscibility of water and PA12 blocks in the extrusion conditions (high pressure and high temperature) makes the dispersion of clay platelets easier, as confirmed by transmission electron microscopy, X-ray diffraction and dynamic rheology. Moreover matrix degradation during extrusion, characterized by gel permeation chromatography, is reduced that way.

Injection of water into the extruder led to exfoliation and intercalation whatever the type of montmorillonite (pristine, i.e. unmodified, or organo-modified). Interestingly, water-assisted compounding is however clearly more efficient in the case of unmodified montmorillonite as the counterpart material obtained without water injection is a microcomposite (presence of micrometric aggregates).

Dynamic mechanical analysis in tension and tensile tests further confirmed the efficiency of water injection. Actually, the modulus of unmodified montmorillonite filled PEBA is higher when compounded with the aid of water and close to the one of organomodified montmorillonite filled PEBA. Furthermore, the dispersion degree has a strong effect on the deformation mechanism and thus on the ductility of the nanocomposites. Indeed, crazes act as energy dissipaters in the case of exfoliated structure but as crack initiators for intercalated nanocomposites. Surprisingly, the ductility of the microcomposite is improved due to the formation of microvoids inside the tactoids, which toughens the material.

To conclude, water-assisted extrusion appears to be an efficient method to elaborate partially exfoliated PEBA/montmorillonite nanocomposites. Particularly, this method allows achieving partial exfoliation of unmodified montmorillonite leading to a significant improvement of the resulting mechanical properties. Thereby, water-assisted melt com- pounding is a convenient alternative to the classical method having recourse to expensive clay organomodification to fabricate nanocomposites based on cheaper unmodified clay such as pristine montmorillonite.

\section{Acknowledgements}

Authors are indebted to the Nord Pas de Calais regional council for facilitating the exchanges between the two collaborating laboratories under international mobility grant \# 2008_09396.

\section{References}

[1] Okada A., Fukushima Y., Kawasumi M., Inagaki S., Usuki A., Sugiyama S., Kurauchi T., Kamigaito O.: Composite material and process for manufacturing same. U.S. Patent 4739007, USA (1988).

[2] Shelley J. S., Mather P. T., DeVries K. L.: Reinforcement and environmental degradation of nylon-6/clay nanocomposites. Polymer, 42, 5849-5858 (2001). DOI: $10.1016 / \mathrm{S} 0032-3861(00) 00900-9$

[3] Liu T., Lim K. P., Tjiu W. C., Pramoda K. P., Chen ZK.: Preparation and characterization of nylon 11/organoclay nanocomposites. Polymer, 44, 3529-3535 (2003). DOI: $10.1016 / \mathrm{S} 0032-3861(03) 00252-0$

[4] Wilkinson A. N., Man Z., Stanford J. L., Matikainen P., Clemens M. L., Lees G. C., Liauw C. M.: Tensile properties of melt intercalated polyamide 6 - Montmorillonite nanocomposite. Composites Science and Technology, 67, 3360-3368 (2007). DOI: 10.1016/j.compscitech.2007.03.024

[5] Alexandre B., Langevin D., Médéric P., Aubry T., Couderc H., Nguyen Q. T., Saiter A., Marais S.: Water barrier properties of polyamide 12/montmorillonite nanocomposite membranes: Structure and volume fraction effects. Journal of Membrane Science, 328, 186204 (2009).

DOI: $10.1016 /$ j.memsci.2008.12.004

[6] Fermeglia M., Ferrone M., Pricl S.: Computer simulation of nylon-6/organoclay nanocomposites: Prediction of the binding energy. Fluid Phase Equilibria, 212, 315-329 (2003). DOI: $10.1016 / \mathrm{S} 0378-3812(03) 00273-5$

[7] Tanaka G., Goettler L. A.: Predicting the binding energy for nylon 6,6/clay nanocomposites by molecular modeling. Polymer, 43, 541-553 (2002). DOI: $10.1016 / \mathrm{S} 1089-3156(01) 00014-9$

[8] Liu A., Xie T., Yang G.: Synthesis of exfoliated monomer casting polyamide $6 / \mathrm{Na}^{+}$-montmorillonite nanocomposites by anionic ring opening polymerization. Macromolecular Chemistry and Physics, 207, 701-707 (2006).

DOI: $\underline{10.1002 / \mathrm{macp} .200500556}$ 
[9] Maiti P., Okamoto M.: Crystallization controlled by silicate surfaces in nylon 6-clay nanocomposites. Macromolecular Materials and Engineering, 288, 440445 (2003).

DOI: $10.1002 /$ mame.200390040

[10] Monticelli O., Musina Z., Frache A., Bellucci F., Camino G., Russo S.: Influence of compatibilizer degradation on formation and properties of PA6/organoclay nanocomposites. Polymer Degradation and Stability, 92, 370-378 (2007).

DOI: $10.1016 /$ j.polymdegradstab.2006.12.010

[11] Zhang F., Low P. F., Roth C. B.: Effects of monovalent, exchangeable cations and electrolytes on the relation between swelling pressure and interlayer distance in montmorillonite. Journal of Colloid and Interface Science, 173, 34-41 (1995).

DOI: $10.1006 /$ jcis. 1995.1293

[12] Kadlecová Z., Puffr R., Baldrian J., Schmidt P., Roda J., Brožek J.: Homoionic inorganic montmorillonites as fillers for polyamide 6 nanocomposites. European Polymer Journal, 44, 2798-2806 (2008).

DOI: $10.1016 /$ j.eurpolymj.2008.06.036

[13] Kim N. H., Malhotra S. V, Xanthos M.: Modification of cationic nanoclays with ionic liquids. Microporous and Mesoporous Materials, 96, 29-35 (2006).

DOI: $10.1016 /$ j.micromeso.2006.06.017

[14] Gilman J. W., Awad W. H., Davis R. D., Shields J., Harris R. H., Davis C., Morgan A. B., Sutto T. E., Callahan J., Trulove P. C., DeLong H. C.: Polymer/ layered silicate nanocomposites from thermally stable trialkylimidazolium-treated montmorillonite. Chemistry of Materials, 14, 3776-3785 (2002).

DOI: $10.1021 / \mathrm{cm} 011532 \mathrm{x}$

[15] Célini N., Bergaya F., Poncin-Epaillard F.: Grafting of hydrocarbon moieties on smectites by cold acetylene plasma and characterization of plasma-treated clay mineral polyethylene nanocomposites. Polymer, 48, 58-67 (2007).

DOI: $10.1016 /$ j.polymer.2006.11.018

[16] Manke C. W., Gulari E., Mielewski D. F., Lee E. C.: System and method of delaminating a layered silicate material by supercritical fluid treatment. U.S. Patent 6469073, USA (2002).

[17] Tomasko D. L., Han X., Liu D., Gao W.: Supercritical fluid applications in polymer nanocomposites. Current Opinion in Solid State and Materials Science, 7, 407412 (2003).

DOI: $10.1016 /$ j.cossms.2003.10.005

[18] Rastogi S., Terry A. E., Vinken E.: Dissolution of hydrogen-bonded polymers in water: A study of nylon4,6. Macromolecules, 37, 8825-8828 (2004). DOI: $10.1021 / \mathrm{ma} 0483423$

[19] Vinken E., Terry A. E., Hoffmann S., Vanhaecht B., Koning C. E., Rastogi S.: Influence of hydrogen bonding on the conformational changes, the brill transition, and lamellae thickening in (co)polyamides. Macromolecules, 39, 2546-2552 (2006).

DOI: $\underline{10.1021 / \mathrm{ma} 0526903}$
[20] Vinken E., Terryt A. E., van Asselen O., Spoelstra A. B., Graf R., Rastogi S.: Role of superheated water in the dissolution and perturbation of hydrogen bonding in the crystalline lattice of polyamide 4,6. Langmuir, 24, 6313-6326 (2008).

DOI: $10.1021 / 1 \mathrm{a} 800378 \mathrm{c}$

[21] Vinken E., Terry A. E., Spoelstra A. B., Koning C. E., Rastogi S.: Influence of superheated water on the hydrogen bonding and crystallography of piperazine-based (co)polyamides. Langmuir, 25, 5294-5303 (2009).

DOI: 10.1021/la804046r

[22] Wevers M. G. M., Mathot V. B. F., Pijpers T. F. J., Goderis B., Groeninckx G.: Full dissolution and crystallization of PA6 and polyamide 4.6 in water and ethanol. in 'Lecture notes in physis' (eds.: Reiter G., Strobl G.) Springer, Berlin, Vol 714, 151-168 (2007).

[23] Wevers M. G. M., Pijpers T. F. J., Mathot V. B. F.: The way to measure quantitatively full dissolution and crystallization of polyamides in water up to $200^{\circ} \mathrm{C}$ and above by DSC. Thermochimica Acta, 453, 67-71 (2007).

DOI: $10.1016 /$ j.tca.2006.10.020

[24] Charlet K., Mathot V., Devaux J.: Crystallization and dissolution behaviour of polyamide 6-water systems under pressure. Polymer International, 60, 119-125 (2011).

DOI: $10.1002 /$ pi.2920

[25] Hasegawa N., Okamoto H., Kato M., Usuki A., Sato $\mathrm{N}$.: Nylon $6 / \mathrm{Na}-$ montmorillonite nanocomposites prepared by compounding nylon 6 with $\mathrm{Na}-$ montmorillonite slurry. Polymer, 44, 2933-2937 (2003).

DOI: 10.1016/S0032-3861(03)00215-5

[26] Fedullo N., Sorlier E., Sclavons M., Bailly C., Lefebvre J-M., Devaux J.: Polymer-based nanocomposites: Overview, applications and perspectives. Progress in Organic Coatings, 58, 87-95 (2007).

DOI: $10.1016 /$ j.porgcoat.2006.09.028

[27] Korbee R. A., Van Geenen A. A.: Process for the preparation of a polyamide nanocomposite composition. U.S. Patent 6350805, USA (1999).

[28] Yu Z-Z., Hu G-H., Varlet J., Dasari A., Ma Y-W.: Water-assisted melt compounding of nylon-6/pristine montmorillonite nanocomposites. Journal of Polymer Science Part B: Polymer Physics, 43, 1100-1112 (2005). DOI: $10.1002 /$ polb.20397

[29] Siengchin S., Karger-Kocsis J., Thomann R.: Nanofilled and/or toughened POM composites produced by water-mediated melt compounding: Structure and mechanical properties. Express Polymer Letter, 2, 746756 (2008).

DOI: $10.3144 /$ expresspolymlett.2008.88

[30] Rubio J., Kitchener J. A.: The mechanism of adsorption of poly(ethylene oxide) flocculant on silica. Journal of Colloid and Interface Science, 57, 132-142 (1976).

DOI: $10.1016 / 0021-9797(76) 90182-\mathrm{X}$ 
[31] Yuang P-C., Shen Y-H.: Determination of the surface area of smectite in water by ethylene oxide chain adsorption. Journal of Colloid and Interface Science, 285, 443-447 (2005).

DOI: $10.1016 /$ j.jcis.2004.12.056

[32] Mathur S., Moudgil B. M.: Adsorption mechanism(s) of poly(ethylene oxide) on oxide surfaces. Journal of Colloid and Interface Science, 196, 92-98 (1997). DOI: $10.1006 /$ jcis. 1997.5192

[33] Su C-C., Shen Y-H.: Adsorption of poly(ethylene oxide) on smectite: Effect of layer charge. Journal of Colloid and Interface Science, 332, 11-15 (2009). DOI: $10.1016 /$ j.jcis.2008.12.024

[34] Strawhecker K. E., Manias E.: Crystallization behavior of poly(ethylene oxide) in the presence of $\mathrm{Na}^{+}$ montmorillonite fillers. Chemistry of Materials, 15, 844-849 (2003).

DOI: $10.1021 / \mathrm{cm} 0212865$

[35] Lu Y., Kong S-T., Deiseroth H-J., Mormann W.: Structural requirements for the intercalation of polyether polyols into sodium-montmorillonite: The role of oxyethylene sequences. Macromolecular Materials and Engineering, 293, 900-906 (2008).

DOI: $10.1002 /$ mame.200800155

[36] Soulestin J.: Elaboration and mechanical behavior of PMMA nanocomposites (in French). PhD thesis. Université des Sciences et Technologies de Lille (2004).

[37] Moad G., Dean K., Edmond L., Kukaleva N., Li G., Mayadunne R. T. A., Pfaendner R., Schneider A., Simon G. P., Wermter H.: Non-ionic, poly(ethylene oxide)-based surfactants as intercalants/dispersants/ exfoliants for poly(propylene)-clay nanocomposites. Macromolecular Materials and Engineering, 291, 3752 (2006).

DOI: 10.1002/mame.200500294

[38] Carretero-González J., Valentín J. L., Arroyo M., Saalwächter K., Lopez-Manchado M. A.: Natural rubber/ clay nanocomposites: Influence of poly(ethylene glycol) on the silicate dispersion and local chain order of rubber network. European Polymer Journal, 44, 3493 3500 (2008).

DOI: 10.1016/j.eurpolymj.2008.08.046

[39] Majumdar D., Dontula N., Blanton T. N., Freedman G. S.: Smectite clay intercalated with polyether block polyamide copolymer. U.S. Patent 7166656, USA (2007).

[40] Yang I-K., Tsai P-H.: Intercalation and viscoelasticity of poly(ether-block-amide) copolymer/montmorillonite nanocomposites: Effect of surfactant. Polymer, 47, 5131-5140 (2006).

DOI: $10.1016 /$ j.polymer.2006.04.065

[41] Yang I-K., Tsai P-H.: Preparation and characterization of polyether-block-amide copolymer/clay nanocomposites. Polymer Engineering and Science, 47, 235243 (2007).

DOI: $10.1002 /$ pen.20670
[42] Sheth J. P., Xu J., Wilkes G. L.: Solid state structureproperty behavior of semicrystalline poly(ether-blockamide) PEBAX ${ }^{\circledR}$ thermoplastic elastomers. Polymer, 44, 743-756 (2003).

DOI: $10.1016 / \mathrm{S} 0032-3861(02) 00798-\mathrm{X}$

[43] Kaufhold S., Dohrmann R., Ufer K., Meyer F. M.: Comparison of methods for the quantification of montmorillonite in bentonites. Applied Clay Science, 22, 145-151 (2002).

DOI: $10.1016 / \mathrm{S} 0169-1317(02) 00131-\mathrm{X}$

[44] Fedullo N., Sclavons M., Bailly C., Lefebvre J-M., Devaux J.: Nanocomposites from untreated clay: A myth? Macromolecular Symposia, 233, 235-245 (2006).

DOI: 10.1002/masy.200690023

[45] Borse N. K., Kamal M. R.: Melt processing effects on the structure and mechanical properties of PA-6/clay nanocomposites. Polymer Engineering and Science, 46, 1094-1103 (2006).

DOI: $10.1002 /$ pen.20578

[46] Tavernier B., Mewis J., Van Puyvelde P., Takenaka M., Ernst B., Hashimoto T.: Effect of thermomechanical history on the crystallization of poly(ether-blockamide). Polymer Engineering and Science, 48, 24182425 (2008).

DOI: $10.1002 /$ pen.21197

[47] Durmus A., Kasgoz A., Macosko C. W.: Linear low density polyethylene (LLDPE)/clay nanocomposites. Part I: Structural characterization and quantifying clay dispersion by melt rheology. Polymer, 48, 4492-4502 (2007).

DOI: 10.1016/j.polymer.2007.05.074

[48] Wagener R., Reisinger T. J. G.: A rheological method to compare the degree of exfoliation of nanocomposites. Polymer, 44, 7513-7518 (2003). DOI: $10.1016 /$ j.polymer.2003.01.001

[49] Wagner W., Cooper J. R., Dittmann A., Kijima J., Kretzschmar H-J., Kruse A., Mareš R., Oguchi K., Sato H., Stöcker I., Šifner O., Takaishi Y., Tanishita I., Trübenbach J., Willkommen T.: The IAPWS industrial formulation 1997 for the thermodynamic properties of water and steam. Journal of Engineering for Gas Turbines and Power, 122, 150-182 (2000).

DOI: $10.1115 / 1.483186$

[50] La Mantia F. P., Scaffaro R.: Melt stabilization of wet polyamide 6. Polymer Degradation and Stability, 75, 473-477 (2002).

DOI: $10.1016 / \mathrm{S} 0141-3910(01) 00250-6$

[51] Levchik S. V., Weil E. D., Lewin M.: Thermal decomposition of aliphatic nylons. Polymer International, 48, 532-557 (1999).

DOI: $10.1002 /(\mathrm{SICI}) 1097-0126(199907) 48: 7<532::$ AIDPI214>3.0.CO;2-R

[52] Soulestin J., Quiévy N., Sclavons M., Devaux J.: Polyolefins-biofibre composites: A new way for an industrial production. Polymer Engineering and Science, 47, 467-476 (2007).

DOI: $10.1002 /$ pen.20706 
[53] Devineau K., Bihannic I., Michot L., Villiéras F., Masrouri F., Cuisinier O., Fragneto G., Michau N.: In situ neutron diffraction analysis of the influence of geometric confinement on crystalline swelling of montmorillonite. Applied Clay Science, 31, 76-84 (2006). DOI: 10.1016/j.clay.2005.08.006

[54] Wan C., Zhao F., Bao X., Kandasuramanian B., Duggan M.: Effect of POSS on crystalline transitions and physical properties of polyamide 12. Journal of Polymer Science Part B: Polymer Physics, 47, 121-129 (2009).

DOI: $10.1002 /$ polb.21620

[55] Abdalla M., Dean D., Adibempe D., Nyairo E., Robinson P., Thompson G.: The effect of interfacial chemistry on molecular mobility and morphology of multiwalled carbon nanotubes epoxy nanocomposite. Polymer, 48, 5662-5670 (2007).

DOI: $10.1016 /$ j.polymer.2007.06.073

[56] Prashantha K., Soulestin J., Lacrampe M. F., Krawczak P., Dupin G., Claes M.: Masterbatch-based multiwalled carbon nanotube filled polypropylene nanocomposites: Assessment of rheological and mechanical properties. Composites Science and Technology, 69, 1756-1763 (2009).

DOI: 10.1016/j.compscitech.2008.10.005

[57] Cassagnau P.: Melt rheology of organoclay and fumed silica nanocomposites. Polymer, 49, 2183-2196 (2008). DOI: $10.1016 /$ j.polymer.2007.12.035

[58] Hoffmann B., Kressler J., Stöppelmann G., Friedrich C., Kim G-M.: Rheology of nanocomposites based on layered silicates and polyamide-12. Colloid and Polymer Science, 278, 629-636 (2000).

DOI: $10.1007 / \mathrm{s} 003960000294$

[59] Li J., Zhou C., Wang G., Zhao D.: Study on rheological behavior of polypropylene/clay nanocomposites. Journal of Applied Polymer Science, 89, 3609-3617 (2003).

DOI: $10.1002 /$ app.12643

[60] Wu D., Zhou C., Zhang M.: Rheology of isothermally crystallized poly(butylene terephthalate) nanocomposites with clay loadings under the percolation threshold. Journal of Polymer Science Part B: Polymer Physics, 45, 229-238 (2007). DOI: $10.1002 /$ polb. 21044

[61] Gagali G., Ramesh C., Lele A.: A rheological study on the kinetics of hybrid formation in polypropylene nanocomposites. Macromolecules, 34, 852-858 (2001). DOI: $10.1021 / \mathrm{ma} 000565 \mathrm{f}$

[62] Dorigato A., Pegoretti A., Penati A.: Linear low-density polyethylene/silica micro- and nanocomposites: Dynamic rheological measurements and modelling. Express Polymer Letters, 4, 115-129 (2010). DOI: $10.3144 /$ expresspolymlett.2010.16
[63] Krishnamoorti R., Yurekli K.: Rheology of polymer layered silicate nanocomposites. Current Opinion in Colloid and Interface Science, 6, 464-470 (2001). DOI: 10.1016/S1359-0294(01)00121-2

[64] Wilkinson A. N., Man Z., Stanford J. L., Matikainen P., Clemens M. L., Lees G. C., Liauw C. M.: Structure and dynamic mechanical properties of melt intercalated polyamide 6-montmorillonite nanocomposites. Macromolecular Materials and Engineering, 291, 917-928 (2006).

DOI: $10.1002 /$ mame.200600150

[65] Varlet J., Cavaillé J. Y., Perez J., Johari G. P.: Dynamic mechanical spectrometry of nylon-12. Journal of Polymer Science Part B: Polymer Physics, 28, 2691-2705 (1990).

DOI: $10.1002 /$ polb.1990.090281315

[66] Huang J-C., Zhu Z-K., Yin J., Qian X-F., Sun Y-Y.: Poly(etherimide)/montmorillonite nanocomposites prepared by melt intercalation: Morphology, solvent resistance properties and thermal properties. Polymer, 42, 873-877 (2001).

DOI: 10.1016/S0032-3861(00)00411-0

[67] Vlasveld D. P. N., Groenewold J., Bersee H. E. N., Picken S. J.: Moisture absorption in polyamide-6 silicate nanocomposites and its influence on the mechanical properties. Polymer, 46, 12567-12576 (2005).

DOI: $10.1016 /$ j.polymer.2005.10.096

[68] Thouzeau C.: Emissions during processing and use of organoclays based nanocomposites. MSc thesis, Université Catholique de Louvain (2009).

[69] Ciprari D., Jacob K., Tannenbaum R.: Characterization of polymer nanocomposite interphase and its impact on mechanical properties. Macromolecules, 39, 65656573 (2006).

DOI: $10.1021 / \mathrm{ma} 0602270$

[70] Kim G-M., Goerlitz S., Michler G. H.: Deformation mechanism of nylon 6/layered silicate nanocomposites: Role of the layered silicate. Journal of Applied Polymer Science, 105, 38-48 (2007).

DOI: 10.1002/app.26067

[71] Cotterell B., Chia J. Y. H., Hbaieb K.: Fracture mechanisms and fracture toughness in semicrystalline polymer nanocomposites. Engineering Fracture Mechanics, 74, 1054-1078 (2007).

DOI: $10.1016 /$ j.engfracmech.2006.12.023

[72] Kim G-M., Lee D-H., Hoffmann B., Kressler J., Stöppelmann G.: Influence of nanofillers on the deformation process in layered silicate/polyamide-12 nanocomposites. Polymer, 42, 1095-1100 (2001). DOI: $10.1016 / \mathrm{S} 0032-3861(00) 00468-7$

[73] He C., Liu T., Tjiu W. C., Sue H-J., Yee A. F.: Microdeformation and fracture mechanisms in polyamide6/organoclay nanocomposites. Macromolecules, 41, 193-202 (2008).

DOI: $10.1021 / \mathrm{ma} 071781 \mathrm{~s}$ 